\title{
Developments of the Pinned Photodiode Terahertz Rectifier
}

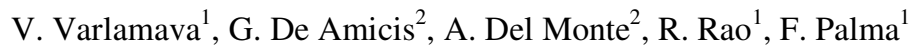 \\ ${ }^{1}$ Department of Information Engineering, Electronics, Telecommunications, Sapienza University of Rome, \\ Rome, Italy, ${ }^{2}$ LFoundry, Avezzano, Italy
}

\begin{abstract}
This paper presents we presents a development of the structure of the pinned photodiode terahertz rectifier, in which the metal whisker of the antenna is separated from the semiconductor by a silane oxide layer, in order to reduce the surface defectiveness.

The rectifies is the basic component of an image detection system based on the structure of actual CMOS image detectors. The structure combines a nano-antenna, fabricated on the top of a standard image sensor, the rectifier, and the readout electronics. The rectifier device proposed has vertical extension of some tenths of nanometers, can be created at the foot of the nano-whisker at the end of the terahertz antenna, above the storage well.
\end{abstract}

$\mathrm{R}$ ECENTLY we presented a new approach to the $\mathrm{THz}$ detectors resulting from the direct integration of the antenna with a rectifying device obtained by modification of commercial CMOS Image Sensors (CMOS ISs) [1].

We demonstrated that a modification of a limited region of the surface of the SW, placed in contact with metal constituting the antenna, results in an efficient rectifying device, with no need to rely on deep submicron technology [2]. The antenna coupled to the rectifying device (a "rectenna" structure) exposed to electromagnetic radiation gives rise to charge injection into the SW, charge that can be collected during the integration time. The rectifying device is designed to ensure the confinement within the SW of the accumulated charge, avoiding leakage currents toward the antenna pad, thus allowing the time integration regime operation.

The sensitivity of the complete system strongly depends on the capability of the rectenna to focus the electric field onto the active region. In order to quantify this capability the field enhancement factor, FE, was used, defined as the ratio between the magnitude of the electric field resulting onto the rectifying device and the magnitude of the incoming electric field. Planar antennas were widely used in previous works, e.g. dipoles, bowties, spirals, log-periodic antennas [3]. We proposed a 3D tapered helix antenna in order to increase the FE on the junction [4].

In this paper we presents an important technological developments in the fabrication of the rectifying device, with an optimization of the metal-semiconductor structure that permits to reduce the defectives of the semiconductor surface and thus to ensure high NEP, and operation in the time integration regime.

\section{REsUlts}

The proposed detector architecture is placed on the backend of the silicon wafer hosting a CMOS IS and represents a combination of an antenna with a nanometric metallic whisker positioned underneath and connecting the antenna to the rectifying device, as shown in Fig. 1 (a).
A dedicated high performance 3D antenna for $\mathrm{THz}$ frequencies fabricated by means of CMOS compatible MEMS technology controlled metal layer release [5] was designed and characterized.

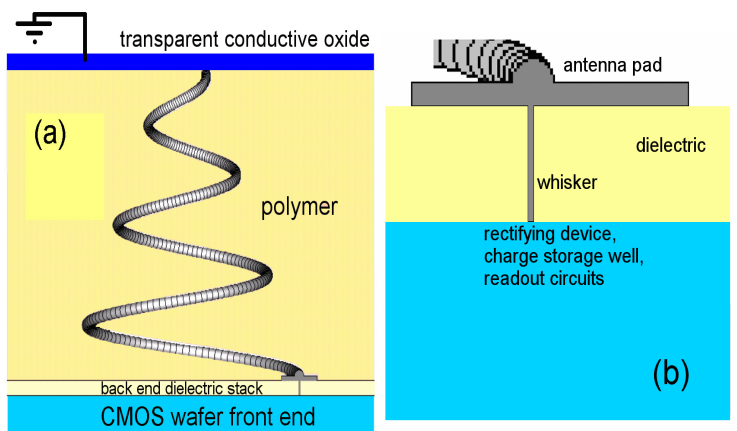

Fig. 1. (a) The geometry of helical tapered conical antenna. (b) Particular of the antenna pad and of the metallic whisker facing the storage well.

The antenna is realized on the top the back-end inter-metal dielectric (IMD) stack of a silicon CMOS wafer containing the IS readout electronics and the rectifying device, and is connected to the latter by means of the bounding pad and a thin metallic whisker crossing the IMD layer, as shown Fig. 1 (b). The structure of the metal whisker is reported in Fig. 2.

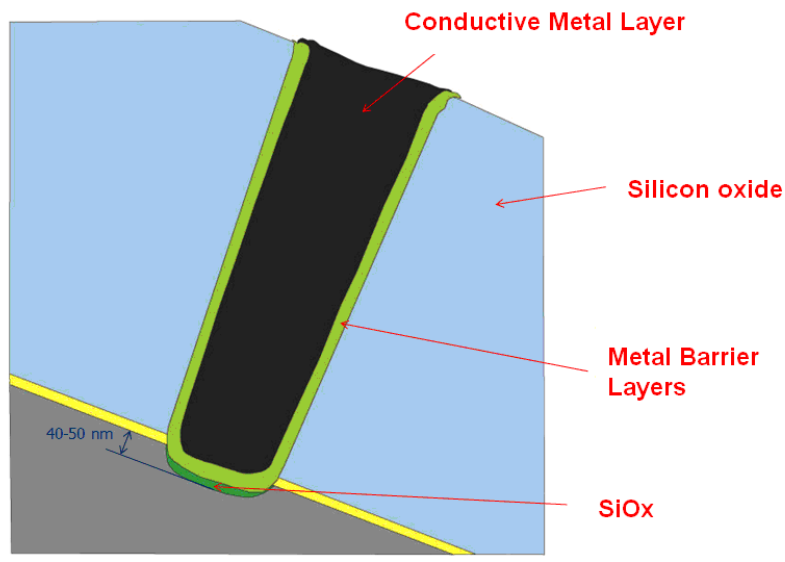

Fig. 2. Schematic section of the standard via contact of CMOS technology, adapted for the $\mathrm{THz}$ rectifier.

The rectifying device adopted in the new structure, wasoptimized from the point of view of the injection of electrons noise in to the SW. The presence of a metal in contact with the silicon surface, beside the $\mathrm{SW}$, indeed introduces a large density of defects, which, extending very close to the storage well, may inject randomly electrons, by thermal generation or by crossing of the barrier by hopping.

A layer of $\mathrm{SiOx}, 70 \AA$ thick, was interposed between the metal and the semiconductor. This material passivates the defects at the semiconductor interface, nevertheless it reduced 
thickness allows penetration of electric field from the antenna. Also the conduction of the rectified current is maintained, by hopping between the defects of the $\mathrm{SiOx}$ layer.

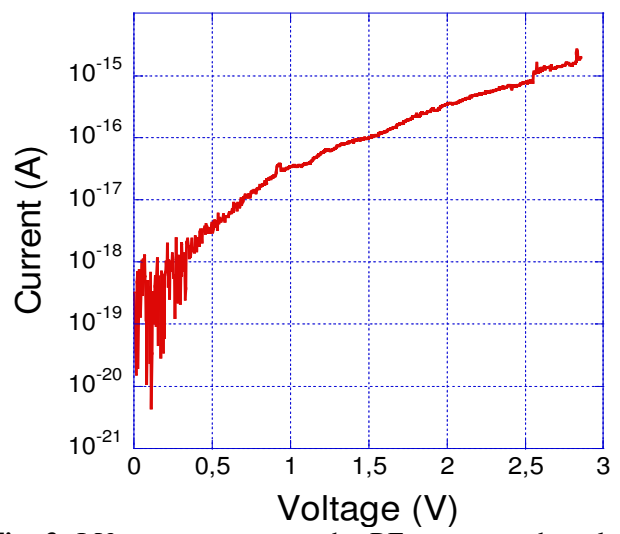

Fig. 3. $\mathrm{I}-\mathrm{V}$ measurements at the RF contact and $\mathrm{p}+$ layer connected to ground on a test structure composed as metal/SiOx/P+silicon

Fig. 3 reports I-V measurements between the whisker contact and p+-Si layer. The test area was a $0.09 \mathrm{~mm} 2$ dot and results were scaled at the dimension of $100 \times 100 \mathrm{~nm}$, the dimension of the whisker base. This measurements shows that the presence of a $\mathrm{SiOx}$ buffer limits the inverse current, maintaining the rectifying capability unaltered. From measured current of Fig. 3, we can evaluate a reverse current lower than 2 electron/s at a forward bias voltage of $100 \mathrm{mV}$ (that is an evaluated ceiling value of forward bias which can be reached by the SW completely emptied of electrons).

The structure was tested at 1,20 , and $35 \mathrm{GHz}$.

RF measurement setup, Agilent E8363B Vector Network Analyzer, was used to measure the input scattering parameter and to apply the RF power to the test structure. At each measurement the RF power was maintained constant and changed step by step along the test.

Fig. 4 reports the rectified current measured by picoammeter versus the RF power applied to the signal pad. As an estimate of the average dark current we adopted the value of 6 electron/s at room temperature measure from the SW.

\section{NEP EVALUATION}

A linear approximation of curves in Fig. 4 was used to evaluate the detector efficiency. Skipping the saturation regime at higher currents, in the low power regime we approximate as linear the relationship between the RF power expressed in $\mathrm{dBm}$, and ten times the logarithm of the current, $\mathrm{I}_{\mathrm{dBA}}$, as given by

$$
P_{d B m}=P_{d B m, 0}+k\left(I_{d B A}-I_{d B A, 0}\right)
$$

where $\mathrm{P}_{\mathrm{dBm}, 0}, \mathrm{I}_{\mathrm{dBA}, 0}$, and the coefficient $\mathrm{k}$ were calculated from geometrical regression of measured data. From the measurement at 1-20-35 GHz we obtained for the three curves a value around $\mathrm{k}=1.18$.

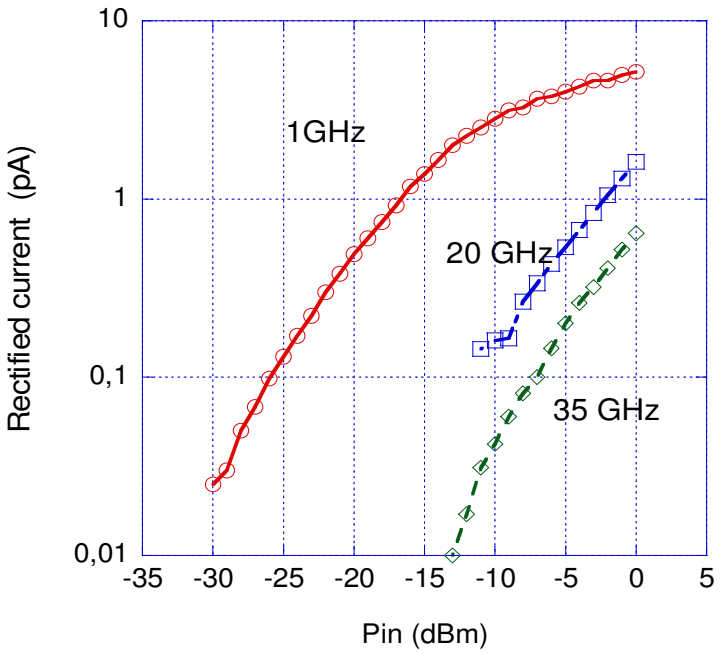

Fig. 4. Rectified current measurement at $1 \mathrm{GHz}$ (red-square curve), $20 \mathrm{GHz}$ (blue-square curve) and $35 \mathrm{GHz}$ (green-diamonds curve).

We followed the NEP evaluation procedure presented in [2]. Numerical simulations of the semiconductor device, performed by means of Synopsis Sentaurus TCAD, showed that the rectification process, experimentally verified, is also effective up to $1 \mathrm{THz}$. One pole behavior, with a cut-off frequency of $120 \mathrm{GHz}$, was identified. We obtained:

$$
N E P(1 T H z)=15.5 p W / \sqrt{H z}
$$

\section{FINAL ABSTRACT}

In this paper a new CMOS compatible direct conversion $\mathrm{THz}$ radiation detector operating at room-temperature is presented and experimentally evaluated. We demonstrate that a layer of $\mathrm{SiOx}, 70 \AA$ thick, interposed between the metal and the semiconductor, which passivates the defects at the semiconductor, does not degrade the detection capability. The rectifier still shows a very high nonlinearity, and gives rise to charge injection into the storage well. Overall performances in terms of noise equivalent power were evaluated by on-wafer RF measurements.

\section{REFERENCES}

[1] Del Monte, F. Palma, "Rettificatore per un Sensore di Radiazioni in Banda Thz, in Particolare per Formazione di Immagini, e Sistema di Raccolta di Carica Comprendente Detto Rettificatore”, Italian Patent, 19 Giugno 2014, No. RM2014A000323.

[2] V. Varlamava, G. De Amicis, A. Del Monte, S. Perticaroli, R. Rao, F. Palma, "CMOS Compatible Room-Temperature Rectifier toward a Terahertz Radiation Detector", Journal of Infrared, Millimeter, and Terahertz Waves, 2016, 1-16.

[3] Jha, Kumud Ranjan, Singh, Ghanshyam, "Terahertz Planar Antennas for Next Generation Communication”, Springer eBooks, 2014

[4] Varlamava, V.; Palma, F.; Nenzi, P.; Balucani, M., "Electric Field Enhancement in 3-D Tapered Helix Antenna for Terahertz Applications" Terahertz Science and Technology, IEEE Transactions on, vol.4, no.3, pp. 360-367, May 2014, DOI 10.1109/TTHZ.2014.2310122

[5] Nenzi, P.; Tripaldi, F.; Varlamava, V.; Palma, F.; Balucani, M., "Onchip THz 3D antennas,"Electronic Components and Technology Conference (ECTC), 2012 IEEE 62nd, pp.102-108, May 29 - June 1, 2012 